\title{
Suvorexant: A boon for Sleepless Nights
}

\author{
Anjan Khadka ${ }^{1}$, Dick Brashier ${ }^{2}$, Amol Vijay Khanapure ${ }^{2}$, Pem Chuki
}

${ }^{1}$ Department of Pharmacology, Nepalese Army Institute of Health Sciences, Nepal. ${ }^{2}$ Department of Pharmacology, Armed Forces Medical College, Pune-40, Maharashtra, India.

\begin{abstract}
Insomnia is characterized by difficulty in falling asleep, difficulty maintaining sleep, or experiencing nonrestorative sleep. Insomnia is the most common medical complaint in general practice. Low efficacy and various side effects limit the use of existing treatment option. Suvorexant is an orexin receptor antagonist (ORA), first in a new class of drugs in development for the treatment of insomnia. It inhibits the wakefulnesspromoting orexin neurons of the arousal system thereby promoting the natural transition from wakefulness. It also improves sleep onset and sleep maintenance and has a favorable tolerability and limited side-effect profile.

Keywords: insomnia; orexin; suvorexant; sleep.
\end{abstract}

\section{Introduction}

Orexins are neuropeptides synthesized from hypothalamus. There are two types of orexins viz. orexin A and orexin B. Both are endogenous ligand for G-protein coupled receptors. ${ }^{[1]}$

Insomnia persistently affects the quality and quantity of sleep. Currently approved treatments for insomnia primarily target $\gamma$-aminobutyric acid-A (GABA-A) receptor signalling and include benzodiazepines and GABA-A receptor modulators. ${ }^{[2]}$ These drugs are used to address this sleep disorder, but have the potential for side effects such as tolerance and dependence, making them less attractive as maintenance therapy. ${ }^{[1,2]}$ Forward and reverse genetic approaches in animals have implicated orexin signalling (also referred to as hypocretin signalling) in the control of vigilance and sleep/wake states. Screening for orexin receptor antagonists using in vitro and in vivo methods in animals has identified compounds that block one or other of the orexin receptors viz. single orexin receptor antagonists (SORAs) or dual orexin receptor antagonists (DORAs]) respectively. SORAs have primarily been used as probes to further elucidate the roles of the individual orexin receptors, while a number of DORAs have progressed to clinical development as pharmaceutical candidates for insomnia ${ }^{2,3}$. The DORA, almorexant demonstrated significant improvements in a number of clinically relevant sleep parameters in animal models and in patients with insomnia but its development was halted ${ }^{4-6}$. Orexin receptor antagonists potentially represent a targeted, effective and welltolerated new class of medications for insomnia.

Suvorexant is an orexin receptor antagonist. The orexin or hypocretins neuropeptide signalling system is a central promoter of wakefulness ${ }^{1,3}$. Inhibition of orexin receptors is thought to suppress wake drive $e^{4}$. FDA announced the approval of suvorexant on August 13,2014 , for the treatment of insomnia ${ }^{7}$.

\section{Mechanism of action}

Suvorexant is a potent dual orexin receptor antagonist that blocks both orexin receptor- 1 and orexin receptor- 2 . It promotes sleep through inhibition of orexin A and B, neuropeptides that promote wakefulness ${ }^{2,4,5}$.

\section{Correspondence:}

Anjan Khadka

Nepalese Army Institute of Health Sciences, Kathmandu.

Email: anjankhadka14@yahoo.com 


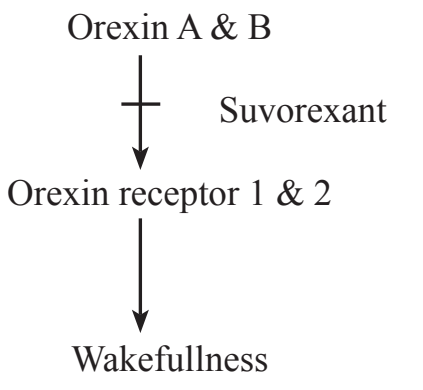

Figure 1: Mechanism of action of suvorexant

\section{Pharmacokinetics}

The onset of action is rapid (56-68 min) with median peak plasma concentrations occur approximately 2 hours after oral administration and are not affected by food. It has volume of distribution of $105.9 \mathrm{~L}$ and is highly protein bound (99.5\%). It is primarily metabolized by cytochrome P450 (CYP3A4) enzyme system. It is eliminated primarily via inactive metabolites in the feces. The half-life is approximately 12.2 hours. Steady state plasma concentrations occur in about three days with daily administration ${ }^{7,8}$.

\section{Clinical efficacy}

The safety and efficacy of suvorexant were based on clinical trials involving more than 500 participants. In the studies, patients taking suvorexant fell asleep faster and spent less time awake during the remainder of the night compared to the patients taking placebo ${ }^{4,9}$. In a randomized, double-blind, placebo controlled trial of suvorexant ranging from 10 to $100 \mathrm{mg}$ involving 22 healthy young males, there was significant changes in both latency to persistent sleep and waking after sleep onset as well as corresponding increase in sleep efficiency and choice reaction time ${ }^{5}$.

In another randomized double blind placebo controlled multicentric trial, suvorexant was found to improved sleep efficacy over four weeks in non elderly adults with primary insomnia, efficacy was assessed primarily via polysomnography (PSG) measurements along with morning and evening questionnaires, administered via an electronic patient diary to record patient's selfassessments of sleep ${ }^{2}$.

\section{Precautions \& Contraindication}

There is no such contraindication to the use of suvorexant although not to be used in narcolepsy and compromised respiratory function ${ }^{7,8}$. Precautions should be taken in conditions like abnormal thinking and behavioral changes, depression, hallucinations and sleep paralysis ${ }^{8}$. There is risk of impaired alertness and motor coordination, including impaired driving ${ }^{10-12}$.

\section{Adverse effects}

The most frequent adverse effects are somnolence $(>5 \%)$, drowsiness and fatigue. Other occasional adverse effects are dizziness, headache, elevated aminotransferase levels, upper respiratory tract infection, etc $\mathrm{et}^{7,12,13}$.

\section{Dosing}

Recommended dose is $10 \mathrm{mg}$ per oral once daily at bed time within 30 minutes of going to bed with at least 7 hours remaining before the planned time of awakening. Dose can be increased maximum upto 20 mg once daily ${ }^{7,12}$.

\section{Drug interactions}

Potent CYP3A inhibitors such as fluconazole increase plasma concentrations of suvorexant. CYP3A inducers such as rifampin result in significantly reduced suvorexant plasma concentrations. Suvorexant is a mild inhibitor of CYP3A, but when administered with CYP3A substrates, including oral contraceptives and warfarin, it had minimal effects ${ }^{8,12,14}$.

\section{CONCLUSION}

Suvorexant is an effective orexin receptor antagonist with a unique clinical profile and the first in a new class of drugs in development for the treatment of insomnia. Though benzodiazepines and non-benzodiazepines are effective for insomnia, their adverse-effect profiles and recommended limitations on long-term use called for other options. Patients who experience both sleep onset and sleep maintenance insomnia may be particularly challenging to treat. The recent approval of orexin antagonist has led to the new era in the treatment of insomnia. 


\section{References}

1. Bennett T, Bray D, Neville MW. Suvorexant, a dual orexin receptor antagonist for the management of insomnia.Pharmacy and Therapeutics. 2014;39 (4):264-6.

2. Herring WJ, Snyder E, Budd K, et al. Orexin receptor antagonism for treatment of insomnia: a randomized clinical trial of suvorexant. Neurology. 2012;79(23):2265-74.

3. Mieda M, Sakurai T. Orexin (hypocretin) receptor agonists and antagonists for treatment of sleep disorders. Rationale for development and current status. CNS Drugs. 2013;27 (2):83-90.

4. Sun H, Kennedy WP, Wilbraham D, Lewis $N$, Calder N, Li X, et al. Effects of suvorexant, an orexin receptor antagonist, on sleep parameters as measured by polysomnography in healthy men. Sleep. 2013;36(2):259-267.

5. Sun H, Kennedy D, Lewis N,Laethem T, Yee K, $\mathrm{Li} X$, et al. The single dose pharmacokinetic $(P K)$ and pharmacodynamic (PD) profiles of suvorexant (MK-4305); a dual orexin receptor antagonist, in healthy male subjects. Clinical Pharmacology and Therapeutics. 2012;91(suppl 1):S29. Available from- http://hdl.handle. net/1854/LU-3129403

6. Neubauer DN. Almorexant, a dual orexin receptor antagonist for the treatment of insomnia. CurrOpinInvestig Drugs. 2010;11(1):101-10.

7. Yang LP. Suvorexant: Fist Global Approval. Drugs. 2014;74(15):1817-22. doi: 10.1007/ s40265-014-0294-5.
8. Hoyer D, Jacobson LH. Orexin in sleep, addiction and more: is the perfect insomnia drug at hand? Neuropeptides. 2013;47(6):477-88. doi: 10.1016/j.npep.2013.10.009.

9. McGaughey $G$, Bayly CI, Cox CD,Schreier $J D$, Breslin MJ, Bogusky $M$, et al. Shaping suvorexant: application of experimental and theoretical methods for driving synthetic designs. J Comput Aided Mol Des. 2014;28:5-12.doi: 10.1007/s10822-014-9710-x.

10. Gotter AL, Winrow CJ, Burner J, Garson SL, Fox $S V$, Binns J, et al. The duration of sleep promoting efficacy by dual orexin receptor antagonists is dependent upon receptor occupancy threshold. BMC Neuroscience. 2013;14:90-116.

11. Nishino S, Ripley B, Overeem S, Lammers GJ, Mignot E. Hypocretin (orexin) deficiency in human narcolepsy. Lancet. 2000;355(9197):3940.

12. Traynor K. Suvorexant approved for insomnia. Am J Health Syst Pharm. 2014;71(18):1524. doi: 10.2146/news 140064.

13. Riemann D, Spiegelhalder K. Orexin receptor antagonists: a new treatment for insomnia? Lancet Neurol. 2014;13(5):441-3. doi: 10.1016/ S1474-4422(13)70311-9.

14. Winrow CJ, Renger JJ. Discovery and development of orexin receptor antagonists as therapeutics for insomnia.Br $J$ Pharmacol. 2014;171(2):283-93. 